M. F. Dahl, A geometric interpretation of the complex tensor Riccati equation for Gaussian beams, Journal of Nonlinear Mathematical Physics, Vol. 14, No. 1, pp. 95-111, 2007.

(C) 2007 by author 


\title{
A geometric interpretation of the complex tensor Riccati equation for Gaussian beams
}

$M F D A H L$

Institute of Mathematics, Helsinki University of Technology, Box 1100, FIN-02015, Finland

E-mail: matias.dahl@tkk.fi

Received May 24, 2006; Accepted in Revised Form August 24, 2006

\begin{abstract}
We study the complex Riccati tensor equation

$$
D_{\dot{c}} G+G C G-R=0
$$

on a geodesic $c$ on a Riemannian 3-manifold. This non-linear equation appears in the study of Gaussian beams. Gaussian beams are asymptotic solutions to hyperbolic equations that at each time instant are concentrated around one point in space. When time moves forward, Gaussian beams move along geodesics, and the Riccati equation determines the Hessian of the phase function for the Gaussian beam. The imaginary part of a solution $G$ describes how a Gaussian beam decays in different directions of space. The main result of the present work is that the real part of $G$ is the shape operator of the phase front for the Gaussian beam. This result generalizes a known result for the Riccati equation in $\mathbb{R}^{3}$. The idea of the proof is to express the Riccati equation in Fermi coordinates adapted to the underlying geodesic. In Euclidean geometry we also study when the phase front is contained in the area of influence, or light cone.
\end{abstract}

\section{Introduction}

We shall study the following equation:

Suppose $c$ is a geodesic parametrized with respect to pathlength in a Riemannian manifold. The complex tensor Riccati equation for tensor $G=\left.G_{i j}(t) d x^{i} \otimes d x^{j}\right|_{c(t)}$ on $c$ is the equation

$$
\begin{aligned}
D_{\dot{c}} G+G C G-R & =0, \\
G(0) & =G_{0},
\end{aligned}
$$

where tensors $C=C^{i j} \frac{\partial}{\partial x^{i}} \otimes \frac{\partial}{\partial x^{j}}$ and $R=R_{i j} d x^{i} \otimes d x^{j}$ on $c$ are defined as

$$
\begin{aligned}
& C^{i j}(t)=\left(g^{i j}-\dot{c}^{i} \dot{c}^{j}\right)(t), \\
& R_{i j}(t)=\left(R_{i j k}^{m} \dot{c}^{k} g_{m l} \dot{c}^{l}\right)(t),
\end{aligned}
$$

and $G_{0}$ is a symmetric complex initial value with positive definite imaginary part. (Precise terminology is given below.) 
This non-linear equation arises in the study of Gaussian beams. These are asymptotic solutions to Maxwell's equations [5, 9, 10, 11] (or wave equation [12, 13, 16, 17]) or elastic equation [14]) that have a very characteristic feature. Namely, at each time instant the whole energy of the solution is concentrated around one point in space. When time moves forward, the energy concentration traverses a curve in space, but its envelope is always a Gaussian bell curve. Gaussian beams are named after this property. Electromagnetic Gaussian beams are also known as quasi-photons [10]. A historical account on the development of Gaussian beams can be found in [1, 16, 17].

The motivation for studying Gaussian beams is that their propagation is completely determined by a set of ordinary differential equations. This means that it is much more easier to propagate a Gaussian beam than to solve the original equations (say, Maxwell's equations in the time domain). In view of this property they have been used to study the traveltime problem: how long does it take for a signal to travel between two points in a possibly inhomogeneous anisotropic media $[12,13]$. In this setting, the physical media determines the Riemannian metric, and Gaussian beams propagate along geodesics of this metric. What is more, the complex tensor Riccati equation determines the Hessian of the phase function for a Gaussian beam (see Section 2.1).

It is well known that the imaginary part of a solution to the complex Riccati equation describes how the Gaussian beam decays in different directions of space (see Section 2.1). The main result of the present work is Theorem 4.5. It shows that the real part of a solution is essentially the shape operator of the phase front for the Gaussian beam (see Section 2.2). This provides a geometric interpretation of solutions to the complex tensor Riccati equation. Even if the equation is non-linear and the real and complex parts are coupled, both parts have a geometric interpretation. A similar result holds for the real Riccati equation in differential geometry. The shape operator for a family of surfaces determined by a distance function satisfy the real Riccati equation $[7,18]$.

In Euclidean space, the geometric character of the real part of a solution to equation (1.1) is known [4]. However, in this case, the Riccati equation is not a tensor equation, and the shape operator is calculated with respect to the Euclidean geometry and not with respect to the geometry induced by the physical media. The main tool for proving Theorem 4.5 are explicit expressions for Fermi coordinates around a geodesic. These are presented in Section 3, and in Section 4 we study the Riccati equation in these coordinates.

In this work we also study properties of the shape operator for solutions in the Euclidean geometry corresponding to homogeneous media (Section 5). In particular, we study when the phase front is contained in the area of influence (or the light cone) and give a sufficient condition on the initial values for this to hold for large $t$.

\section{Basic definitions}

We shall work in a differential geometric setting and assume that all objects are smooth. By a manifold $M$ we mean a Hausdorff, second countable, topological manifold that has smooth transition maps. The tangent and cotangent bundles of $M$ (over $\mathbb{R}$ ) are denoted by $T M$ and $T^{*} M$, respectively. By $I$ we mean either an open interval containing 0 or the identity matrix. We assume that $M$ is equipped with a Riemannian metric tensor $g$ and locally $g=g_{i j} d x^{i} \otimes d x^{j}$. The Einstein's summation convention is employed throughout. By $g^{i j}$ we mean the $i j$ :th entry of the inverse of $\left(g_{i j}\right)$. Christoffel symbols $\Gamma_{i j k}, \Gamma_{j k}^{i}$ are 
defined as

$$
\begin{aligned}
\Gamma_{i j k} & =\frac{1}{2}\left(\frac{\partial g_{i k}}{\partial x^{j}}+\frac{\partial g_{i j}}{\partial x^{k}}-\frac{\partial g_{j k}}{\partial x^{i}}\right), \\
\Gamma_{j k}^{i} & =g^{i a} \Gamma_{a j k} .
\end{aligned}
$$

A curve $c: I \rightarrow M$ is a geodesic parametrized with respect to pathlength if

$$
\begin{aligned}
\frac{d^{2} c^{i}}{d t^{2}}+\Gamma_{j k}^{i}(t) \dot{c}^{j} \dot{c}^{k} & =0, \\
g_{i j}(t) \dot{c}^{i} \dot{c}^{j} & =1 .
\end{aligned}
$$

As above, we will frequently use notation $g_{i j}(t), \Gamma_{j k}^{i}(t)$, etc., which simply means that $g_{i j}$ and $\Gamma_{j k}^{i}$ are evaluated at $c(t)$. The components of the Riemann curvature tensor $R=R_{i j k}^{m} d x^{i} \otimes d x^{j} \otimes d x^{k} \otimes \frac{\partial}{\partial x^{m}}$ are

$$
R_{i j k}^{m}=\frac{\partial \Gamma_{i k}^{m}}{\partial x^{j}}-\frac{\partial \Gamma_{i j}^{m}}{\partial x^{k}}+\Gamma_{i k}^{s} \Gamma_{j s}^{m}-\Gamma_{i j}^{s} \Gamma_{k s}^{m} .
$$

If $\left(x^{i}, y^{i}\right)$ are local coordinates for $T M$ induced by $x^{i}$-coordinates for $M$, we define

$$
N_{j}^{i}(x, y)=\Gamma_{j k}^{i}(x) y^{k} .
$$

Let us also introduce notation

$$
p_{i}(t)=g_{i j}(t) \dot{c}^{j}(t) .
$$

A complex tensor on a curve $c$ is a mapping that map a point $t \in I$ into a tensor on $M$ at $c(t)$ with possibly complex coefficients (see e.g. [5]). Locally such a tensor $\alpha$ is determined by suitable functions $\alpha_{i_{1} \cdots i_{n}}^{k_{1} \cdots k_{m}}: I \rightarrow \mathbb{C}$, such that

$$
\alpha(t)=\left.\alpha_{i_{1} \cdots i_{n}}^{k_{1} \cdots k_{m}}(t) d x^{i_{1}} \otimes \cdots \otimes d x^{i_{n}} \otimes \frac{\partial}{\partial x^{k_{1}}} \otimes \cdots \otimes \frac{\partial}{\partial x^{k_{m}}}\right|_{c(t)} .
$$

For a tensor $\alpha=\alpha_{i}(t) d x^{i}$, its covariant derivatives along the curve $c$ is defined as

$$
D_{\dot{c}} \alpha=\left.\left(\frac{d \alpha_{i}}{d t}-\alpha_{k} N_{i}^{k} \circ \dot{c}\right) d x^{i}\right|_{c(t)},
$$

and for higher tensors $D(u \otimes v)=D(u) \otimes v+u \otimes D(v)$. By $\mathfrak{X}(M)$ we denote the set of smooth vector fields on $M$. Suppose $X=X^{i} \frac{\partial}{\partial x^{i}} \in \mathfrak{X}(M)$, and $\alpha=\alpha_{i} d x^{i}$ is a 1 -form on $M$. Then for a vector $v=v^{i} \frac{\partial}{\partial x^{i}} \in T M$ we define covariant derivatives

$$
\begin{aligned}
D_{v} X & =v^{k}\left(\frac{\partial X^{i}}{\partial x^{k}}+X^{j} \Gamma_{j k}^{i}\right) \frac{\partial}{\partial x^{i}} \\
D_{v} \alpha & =v^{k}\left(\frac{\partial \alpha_{i}}{\partial x^{k}}-\alpha_{j} \Gamma_{i k}^{j}\right) d x^{i} .
\end{aligned}
$$

By $\operatorname{Re} L, \operatorname{Im} L$ we mean the real and imaginary parts of a tensor or matrix $L$, and $\delta_{i j}, \delta_{i}^{j}$ are Kronecker delta symbols. By $\bar{A}$ we mean the closure of a set $A$. 


\section{Shape operator}

Suppose $S$ is a orientable hypersurface of a manifold $M$, and suppose that $n: S \rightarrow T M$ is a unit normal to $S$. Let $p \in S$, and let $U \subset M$ be a neighbourhood of $p$. By shrinking $U$, we can extend $n$ into a vector field $\hat{n}: U \rightarrow T U$. We may also assume that $g(\hat{n}, \hat{n})=1$ on $U$. For $v \in T_{p} S$, let

$$
L(v)=\left(D_{v} \hat{n}\right)(p) .
$$

Then one can prove that $L(v)$ does not depend on the extension of $n$, and furthermore, $L(v) \in T_{p} S$. The mapping $L: T S \rightarrow T S$ is the shape operator (or Weingarten map) of hypersurface $S$. It is determined up to a sign with respect to $n$. Let us also point out that sometimes the shape operator is defined as $-D_{v}(\hat{n})_{p}[20]$. One can show that the eigenvalues of $L$ are real. On a 3 -manifold, $L$ has 2 eigenvalues $\kappa_{1}, \kappa_{2}$ called principal curvatures. The Gaussian curvature is defined as $K=\kappa_{1} \kappa_{2}$, and the mean curvature is defined as $S=\frac{1}{2}\left(\kappa_{1}+\kappa_{2}\right)$.

\section{Riccati equation for Gaussian beams}

\subsection{Physical background}

Let us briefly describe Gaussian beams and their relation to the complex tensor Riccati equation. Detailed expositions on this topic are $[5,10,11,12,17]$. Let us start with the source-less Maxwell's equations for differential forms

$$
\begin{aligned}
d E & =-\frac{\partial B}{\partial t}, \\
d H & =\frac{\partial D}{\partial t},
\end{aligned}
$$

and let us consider an electric field of the form

$$
E(x, t)=\operatorname{Re}\left\{E_{0}(x, t) \exp (i P \theta(x, t))\right\}, \quad(x, t) \in M \times I .
$$

Here $P>0$ is a large constant, $I$ is an open interval representing time, $E_{0}$ is a complex 1-form, and the function $\theta: M \times I \rightarrow \mathbb{C}$ is the phase function for $E$. The advantage of representing the electric field as above is that qualitatively $E_{0}$ and $\theta$ contain different type of information. The 1-form $E_{0}$ completely determines how $E$ is polarized. For the phase function, $\operatorname{Re} \theta$ describes high frequency oscillations of $E$, that is, information about propagation, while $\operatorname{Im} \theta$ influences the amplitude of $E$. In order for $E$ to be stable in the limit $P \rightarrow \infty$, we assume that $\operatorname{Im} \theta \geq 0$.

Suppose $c: I \rightarrow M$ is a smooth curve. Furthermore, suppose $c$ is covered with local coordinates $x^{i}$, and suppose that

$$
\phi: I \rightarrow \mathbb{C}, \quad p: I \rightarrow \mathbb{C}^{3}, \quad H: I \rightarrow \mathbb{C}^{3 \times 3}
$$

are the first three coefficients in the Taylor expansion of $\theta$ evaluated on $c(t)$. That is,

$$
\phi(t)=\theta(c(t), t), \quad p_{j}(t)=\frac{\partial \theta}{\partial x^{j}}(c(t), t), \quad H_{j k}(t)=\frac{\partial^{2} \theta}{\partial x^{j} \partial x^{k}}(c(t), t) .
$$

Then $E$ is a Gaussian beam on $c$ provided that for all $t \in I$, 
1. $p(t)=\left(p_{i}(t)\right)_{i}$ is non-zero,

2. $\phi(t)$ and $p(t)$ are real,

3. the imaginary part of $H(t)=\left(H_{i j}(t)\right)_{i j}$ is positive definite.

One can also show that these conditions do not depend on local coordinates [5, 12]. Using the chain rule, it follows that [5]

$$
\theta(x, t)=\phi_{0}(t)+p_{i}(t) z^{i}+\frac{1}{2} H_{i j}(t) z^{i} z^{j}+O\left(|z|^{3}\right)
$$

where $z^{i}=z^{i}(x, t)=x^{i}-c^{i}(t)$. In consequence, $|\exp (i P \theta(x, t))| \approx \exp \left(-\frac{P}{2} z^{i} \operatorname{Im} H_{i j} z^{j}\right)$. In other words, at time $t$, the energy of $E$ is completely concentrated around $c(t)$, and Im $H$ describes the shape of the field.

Plugging $E(x, t)$ into Maxwell's equation (see $[5,9,10,11]$ for details) yields the following conditions on $\theta: \phi_{0}$ is constant, $c$ is a geodesic with respect to a suitable Riemannian geometry depending on the media, and $p_{i}(t)$ are given by equation (1.3). Furthermore, if $\Lambda(t)=\left(\Gamma_{i j}^{m} p_{m}\right)_{i j}$, then

$$
G(t)=\left.G_{i j}(t) d x^{i} \otimes d x^{j}\right|_{c(t)}, \quad G_{i j}=(H-\Lambda)_{i j}
$$

is a solution to the complex tensor Riccati equation $[5,12]$. Thus $\operatorname{Im} G=\operatorname{Im} H$ determines how a Gaussian beam decays in different directions from $c(t)$. If we are in isotropic media with permittivity $\varepsilon$ and permeability $\mu$, then Gaussian beams propagate along geodesics of the scaled Euclidean metric [5] $g=\frac{1}{\varepsilon \mu} \delta_{i j} d x^{i} \otimes d x^{j}$. One can also derive equations for the vector part $E_{0}$ in $E$, and these are studied in [10].

\subsection{Phase front surface}

For a fixed $t \in I$, let us define the phase front surface,

$$
F_{t}=\{x \in M: \operatorname{Re} \theta(x, t)=\operatorname{Re} \theta(c(t), t)\} .
$$

(In the proof of Proposition 2.2 we show that $F_{t}$ is a surface.) On $F_{t}$, $\operatorname{Re} \theta$ is constant, that is, $\exp (i P \theta)$ does not oscillate. Thus all oscillations happen through $F_{t}$. For example, for a plane wave, $F_{t}$ would be sheets orthogonal to the direction of propagation. The next two propositions give some geometric information about $F_{t}$. Proposition 2.1 is proved in [3] for real phase functions.

Proposition 2.1. Phase front surface $F_{t}$ is orthogonal to $\dot{c}(t)$ with respect to $g$.

Proof. Let $u \in T_{c(t)} F_{t}$. It follows that $u=\left.\frac{d \gamma^{i}}{d s}(0) \frac{\partial}{\partial x^{i}}\right|_{c(t)}$ for some curve $\gamma:(-\varepsilon, \varepsilon) \rightarrow$ $M$ such that $\gamma(0)=c(t)$ and $s \mapsto \operatorname{Re} \theta(\gamma(s), t)$ is constant. Since $p_{i}(t)=\frac{\partial \theta}{\partial x^{i}}(c(t), t)$, equation (1.3) implies that $g(u, \dot{c})=\left.\frac{d \theta(\gamma(s), t)}{d s}\right|_{s=0}$, and the proposition follows since $g(u, \dot{c})$ is real. 
Proposition 2.2. Let $\hat{F}_{t}$ be the representation of the phase front in local coordinates equipped with the Euclidean inner product. Then the Gaussian curvature $K$ and the mean curvature $S$ of $\hat{F}_{t}$ at $c(t)$ are

$$
\begin{aligned}
K & =\frac{p \cdot \operatorname{adj}(\operatorname{Re} H) \cdot p^{T}}{\|p\|_{0}^{4}}, \\
S & =-\frac{p \cdot \operatorname{Re}(H-\operatorname{trace}(H) I) \cdot p^{T}}{2\|p\|_{0}^{3}},
\end{aligned}
$$

where $p$ is the Euclidean vector $p=\left(p_{1}, p_{2}, p_{3}\right),\|\cdot\|_{0}$ is the Euclidean norm, and adj is the adjugate of a matrix. Furthermore, $p$ is a unit normal to $\hat{F}_{t}$ at $c(t)$, and $K$ and $S$ are calculated with respect to this orientation for $F_{t}$.

Proof. Let us first calculate the principal curvatures $\kappa_{1}, \kappa_{2}$ of $\hat{F}_{t}$ at $c(t)$. Let $f(x)=$ $\operatorname{Re} \theta(x, t)-\operatorname{Re} \theta(c(t), t)$. Then $(\nabla f)(c(t))=p(t)$, and $(\operatorname{Hess} f)(c(t))=\operatorname{Re} H(t)$. Since $p(t) \neq 0, F_{t}$ is a surface. Next, we employ a result from [20, p. 204]: If $s_{1}, s_{2}$ are the two roots to the $4 \times 4$ determinant equation

$$
\operatorname{det}\left(\begin{array}{c|c}
\operatorname{Re} H-s I & p^{T} \\
\hline p & 0
\end{array}\right)=0,
$$

then $\kappa_{i}=\|p\|_{0}^{-1} s_{i}$. (Here we have taken into account that in [20], the shape operator is defined with opposite sign.) Using computer algebra, we find that $s_{i}$ are solutions to $A s^{2}+B s+C=0$, where

$$
\begin{aligned}
& A=-\|p\|_{0}^{2}, \\
& B=-p \cdot \operatorname{Re}(H-\operatorname{trace}(H) I) \cdot p^{T}, \\
& C=-p \cdot \operatorname{adj}(\operatorname{Re} H) \cdot p^{T} .
\end{aligned}
$$

The result follows from Viete's formulas; $s_{1} s_{2}=C / A$ and $s_{1}+s_{2}=-B / A$.

\subsection{Solving the Riccati equation}

Assuming that $G_{0}$ is symmetric and has positive definite imaginary part, one can show that equation (1.1) is well posed. A solution $G(t)$ exists for all $t$ where $c$ is defined, and such a solution is unique. In addition, if $G$ is a Gaussian beam at one $t$, it will be a Gaussian beam for all $t[5,12]$.

Let us next outline the standard way to solve equation (1.1). Namely, how to reduce it into a linear system. For simplicity, let us assume that we can cover geodesic $c$ with some local coordinates, whence we can define matrices $C=\left(C^{i j}\right)_{i j}, G=\left(G_{i j}\right)_{i j}, R=\left(R_{i j}\right)_{i j}$, $N=\left(N_{i}^{j} \circ \dot{c}\right)_{i j}$ all depending on $t$. Let us point out that matrices $C, G, R$ are symmetric (see Section 3.1 or [5]). Equation (1.1) is then equivalent to the matrix equation [5, 12]

$$
G^{\prime}-N G-G N^{T}+G C G-R=0 .
$$

Let $Y, Z$ be the solution to the linear system

$$
\begin{aligned}
\left(\begin{array}{l}
Y \\
Z
\end{array}\right)^{\prime} & =\left(\begin{array}{cc}
0 & I \\
-I & 0
\end{array}\right)\left(\begin{array}{cc}
-R & -N \\
-N^{T} & C
\end{array}\right)\left(\begin{array}{l}
Y \\
Z
\end{array}\right), \\
Y(0) & =I, \quad Z(0)=G_{0} .
\end{aligned}
$$


Using the assumptions on $G_{0}$, one can show that $Y$ is invertible for all $t$. Furthermore, $G=Z Y^{-1}$ is the unique solution to equation (2.1), and so $G$, interpreted as a 2-tensor is a solution to equation (1.1). The next lemma shows that $G$ and $Z$ are invertible for all $t$.

Lemma 2.3. A symmetric complex matrix with positive definite imaginary part is invertible.

Proof. Let $A, B$ be real symmetric matrices, such that $B$ is positive definite. If $\operatorname{det}(A+$ $i B)=0$, then $-i \in \sigma\left(B^{-1} A\right)$ (the spectrum of $\left.B^{-1} A\right)$. Since $B^{-1}$ is positive definite, it has a symmetric square root, whence $-i \in \sigma\left(B^{-1} A\right)=\sigma\left(\sqrt{B^{-1}} A \sqrt{B^{-1}}\right)$. This is a contradiction since $\sqrt{B^{-1}} A \sqrt{B^{-1}}$ is symmetric.

In the lemma below, the first equation states that $\operatorname{Im} G$ is determined by $Y$ alone.

Proposition 2.4. Suppose $Y, Z$ are solutions to equation (2.2). Then

$$
\begin{aligned}
\operatorname{Im} G & =\left(Y^{-1}\right)^{*} \operatorname{Im}\left(G_{0}\right) Y^{-1}, \\
\operatorname{Im}\left(G^{-1}\right) & =-\left(Z^{-1}\right)^{*} \operatorname{Im}\left(G_{0}\right) Z^{-1},
\end{aligned}
$$

where $A^{*}$ is the conjugate transpose of a matrix $A$.

Proof. As $R$ and $C$ are symmetric, equation (2.1) implies that

$$
\frac{d}{d t}\left(\left(\begin{array}{l}
Y \\
Z
\end{array}\right)^{*}\left(\begin{array}{cc}
0 & I \\
-I & 0
\end{array}\right)\left(\begin{array}{l}
Y \\
Z
\end{array}\right)\right)=0
$$

so $Y^{*} Z-Z^{*} Y=G_{0}-G_{0}^{*}$. Since $G$ is symmetric,

$$
\begin{aligned}
\operatorname{Im} G & =\frac{1}{2 i}\left(G-G^{*}\right) \\
& =\frac{1}{2 i}\left(Y^{-1}\right)^{*}\left(Y^{*} Z-Z^{*} Y\right) Y^{-1} \\
& =\frac{1}{2 i}\left(Y^{-1}\right)^{*}\left(G_{0}-G_{0}^{*}\right) Y^{-1} .
\end{aligned}
$$

In particular, $\operatorname{Im} G_{0}=\frac{1}{2 i}\left(G_{0}-G_{0}^{*}\right)$, and the first claim follows. The second claim follows similarly since $G^{-1}$ is symmetric.

\section{$3 \quad$ Fermi coordinates}

Next we construct coordinates around a geodesic such that on the geodesic, the metric tensor is Euclidean and all Christoffel symbols vanish. More generally, one can find local coordinates around any curve such that the Christoffel symbols vanish. Original references to this result by Fermi (1922) and Levi-Civita $(1925-26)$ are given in [6, p. 92].

In Section 3.1 we show that in Fermi coordinates, $N=0$, and $C$ is constant. This will considerably simplify equation (2.1) [2].

Theorem 3.1 (Fermi coordinates). Suppose $M$ is a Riemannian $n$-manifold, $n \geq 2$, and $c: J \rightarrow M$ is a geodesic parametrized with respect to pathlength that does not intersect itself. Furthermore, suppose $c(J)$ is contained in one coordinate chart, and $I$ is an open 
bounded interval satisfying $\bar{I} \subset J$. Then there exist local coordinates $\left(\tilde{x}^{1}, \tilde{x}^{2}, \ldots, \tilde{x}^{n}\right)=$ $\left(t, \mu^{1}, \ldots, \mu^{n-1}\right)$ that cover $c(I)$, and for $t \in I$,

$$
\begin{aligned}
(t, \mu=0) & \text { represents } c(t), \\
\dot{c}(t) & =\left.\frac{\partial}{\partial t}\right|_{c(t)}, \\
\tilde{g}_{i j}(t) & =\delta_{i j}, \\
\frac{\partial \tilde{g}_{i j}}{\partial \tilde{x}^{k}}(t) & =0 \\
\tilde{\Gamma}_{j k}^{i}(t) & =0 .
\end{aligned}
$$

Coordinates $t, \mu^{i}$ as in Proposition 3.1 are called Fermi coordinates. Since the first Fermi coordinate is in a special position, we shall use an adapted Einstein summation convention: for Roman letters the summation range is $1, \ldots, n$, and for Greek letters the range is $2, \ldots, n$.

One way to prove Theorem 3.1 is to construct coordinates $(t, \mu)$ such that for constant $(t, \mu)$, curves $s \mapsto(t, s \mu)$ represent geodesics [15]. The disadvantage, however, with this construction is that it gives the coordinates in a rather implicit form. The proof below is less geometric, but the coordinates are given explicitly by equation (3.6). This expression will be needed in Section 4 and in the proof of Theorem 4.5. Let us also emphasize that the below Fermi coordinates are global (up the the endpoints).

Proof of Theorem 3.1. Let $x^{i}$ be coordinates that cover $c(J)$. For some $t_{0} \in I$, let vectors $P_{2}\left(t_{0}\right), \ldots, P_{n}\left(t_{0}\right)$ be such that $\left\{\dot{c}\left(t_{0}\right), P_{2}\left(t_{0}\right), \ldots, P_{n}\left(t_{0}\right)\right\}$ form an orthonormal basis. For a general $t \in J$, let $P_{\alpha}(t)$ be the the parallel transport of $P_{\alpha}\left(t_{0}\right)$ along $c$ to $c(t)$. In other words,

$$
P_{\alpha}(t)=\left.P_{\alpha}^{i}(t) \frac{\partial}{\partial x^{i}}\right|_{c(t)}, \quad \alpha=2, \ldots, n,
$$

where coefficients $P_{\alpha}^{i}(t)$ are solutions to ordinary differential equations $\dot{P}_{\alpha}^{i}+N_{s}^{i}(t) P_{\alpha}^{s}=0$. For $\tilde{x}^{i}=\left(t, \mu^{2}, \ldots, \mu^{n}\right) \in J \times \mathbb{R}^{n-1}$, let us define a map $\tilde{x} \mapsto x(\tilde{x})$ by

$$
\begin{aligned}
x^{i}(\tilde{x}) & =c^{i}(t)-P_{\alpha}^{i}(t) \mu^{\alpha}-\frac{1}{2} Q_{\alpha \beta}^{i}(t) \mu^{\alpha} \mu^{\beta}, \\
Q_{\alpha \beta}^{i} & =\Gamma_{a b}^{i} P_{\alpha}^{a} P_{\beta}^{b} .
\end{aligned}
$$

Let $B_{r}$ be the Euclidean open ball in $\mathbb{R}^{n-1}$ with center 0 and radius $r>0$. For some $r>0, x\left(\bar{I} \times B_{r}\right)$ is completely contained in $x^{i}$-coordinates. (Proof: Let $U$ be the chart for the $x^{i}$ coordinates. Suppose that for each $l=1,2, \ldots$, there exists an $y_{l} \in x\left(\bar{I} \times \bar{B}_{1 / l}\right)$ such that $y_{l} \notin U$. On a subsequence, $y_{l} \rightarrow c(a)$ for some $a \in \bar{I}$. This contradicts $y_{l} \notin U$ since $c(a) \in c(\bar{I}) \subset c(J) \subset U$, and $U$ is open.)

The rest of the proof is divided into two steps. First we show that $\tilde{x}^{i}$-coordinates are coordinates. Thereafter we show that the coordinates satisfy the sought conditions. 
Step 1. Since the parallel transport preserves the inner product, $\left\{\dot{c}(t), P_{2}(t), \ldots, P_{n}(t)\right\}$ are orthonormal for all $t \in J$ and, in particular, linearly independent. Since

$$
\begin{aligned}
\frac{\partial x^{i}}{\partial t}(t, \mu) & =\dot{c}^{i}+N_{s}^{i}(t) P_{\alpha}^{s} \mu^{\alpha}-\frac{1}{2} \dot{Q}_{\alpha \beta}^{i} \mu^{\alpha} \mu^{\beta}, \\
\frac{\partial x^{i}}{\partial \mu^{\alpha}}(t, \mu) & =-P_{\alpha}^{i}-Q_{\alpha \beta}^{i} \mu^{\beta},
\end{aligned}
$$

the Jacobian of $\tilde{x} \mapsto x(\tilde{x})$ is pointwise invertible on $\bar{I} \times\{0\}$. It follows that there exists an open set $X \subset J \times B_{r}$ such that $x: X \rightarrow x(X)$ is locally a diffeomorphism. Lemma 9.19 in [19] implies that for some $r>0, x: I \times B_{r} \rightarrow x\left(I \times B_{r}\right)$ is a diffeomorphism.

Step 2. Equations (3.1) and (3.2) follow from equation (3.6). Also, by orthogonality, $g_{i j}(t) P_{\alpha}^{i} P_{\beta}^{j}=\delta_{\alpha \beta}, g_{i j}(t) P_{\alpha}^{i} \dot{c}^{j}=0, g_{i j}(t) \dot{c}^{i} \dot{c}^{j}=1$. Equation (3.3) now follows from $\tilde{g}_{i j}=$ $\frac{\partial x^{a}}{\partial \tilde{x}^{i}} \frac{\partial x^{b}}{\partial \tilde{x}^{j}} g_{a b}$, and equation (3.5) follows from

$$
\tilde{\Gamma}_{i j k}=\frac{\partial x^{a}}{\partial \tilde{x}^{i}}\left(\frac{\partial^{2} x^{b}}{\partial \tilde{x}^{j} \partial \tilde{x}^{k}} g_{a b}+\frac{\partial x^{b}}{\partial \tilde{x}^{j}} \frac{\partial x^{c}}{\partial \tilde{x}^{k}} \Gamma_{a b c}\right) .
$$

Here it suffices to show that the parenthesis vanishes for all $j, k$. Equation (3.4) follows from the identity $\frac{\partial g_{i j}}{\partial x^{k}}=\Gamma_{i j k}+\Gamma_{j i k}$.

\subsection{Curvature}

In Fermi coordinates, the Riemann curvature tensor takes the form

$$
\tilde{R}_{i j k}^{m}(t, \mu)=\frac{1}{2}\left(\frac{\partial^{2} \tilde{g}_{m k}}{\partial \tilde{x}^{i} \partial \tilde{x}^{j}}-\frac{\partial^{2} \tilde{g}_{i k}}{\partial \tilde{x}^{m} \partial \tilde{x}^{j}}+\frac{\partial^{2} \tilde{g}_{i j}}{\partial \tilde{x}^{m} \partial \tilde{x}^{k}}-\frac{\partial^{2} \tilde{g}_{m j}}{\partial \tilde{x}^{i} \partial \tilde{x}^{k}}\right) .
$$

This follows by inserting $\tilde{\Gamma}_{i k}^{m}=\tilde{g}^{m s} \tilde{\Gamma}_{s i k}$ into equation (1.2), differentiating, using $\tilde{g}_{i j}=\delta_{i j}$, $\tilde{\Gamma}_{j k}^{i}=0$, and the definition of $\Gamma_{i j k}$. (At the expansion point in normal coordinates, the Riemann curvature tensor satisfies the same formula. See [8, p. 130])

In Fermi coordinates, tensors $C$ and $R$ have matrix representations

$$
\begin{aligned}
\tilde{C} & =\operatorname{diag}(0,1,1), \\
\tilde{R} & =\operatorname{diag}(0, \mathscr{R}),
\end{aligned}
$$

where $\mathscr{R}: I \rightarrow \mathbb{R}^{2 \times 2}$ is a $2 \times 2$ symmetric matrix. The latter claim follows since $\tilde{R}_{1 i}=$ $\tilde{R}_{i 1}=0$ and $\tilde{R}_{i j}=\tilde{R}_{i j 1}^{1}$, as can be seen from equation (3.8). Furthermore, as $\tilde{\Gamma}_{j k}^{i}=0$ we have $\tilde{N}_{j}^{i}=0$.

\section{Gaussian beams in Fermi coordinates}

Definition 1 (Surface $S_{t}$ ). For a fixed $t$, let $S_{t}$ be the Fermi coordinate surface $t=$ constant passing through $c(t)$.

On $S_{t}$, a second order approximation of $\theta$ is

$$
\begin{aligned}
\theta(x(t, \mu), t) & \approx \phi_{0}+\frac{1}{2} G_{j k} P_{\alpha}^{j} \mu^{\alpha} P_{\beta}^{k} \mu^{\beta} \\
& =\phi_{0}+\frac{1}{2} \tilde{G}_{\alpha \beta}(t) \mu^{\alpha} \mu^{\beta} .
\end{aligned}
$$

This shows that on $S_{t}$ a Gaussian beam decays approximately as $\exp \left(-\frac{P}{2} \operatorname{Im} \tilde{G}_{\alpha \beta} \mu^{\alpha} \mu^{\beta}\right)$. 


\subsection{Decomposition of the Riccati equation}

For matrices $\tilde{G}, \tilde{C}, \tilde{R}$ representing tensors $G, C, R$ in Fermi coordinates, equation (2.1) reads

$$
\tilde{G}^{\prime}+\tilde{G} \tilde{C} \tilde{G}-\tilde{R}=0 .
$$

In other words, when we formulate the Riccati equation in Fermi coordinates, two terms vanish, and $\tilde{C}$ becomes a constant matrix. In addition, since matrices $\tilde{C}, \tilde{R}$ are non-zero only in their lower $2 \times 2$ blocks, it turns out that equation (4.1) is only a $2 \times 2$ Riccati equation (equation (4.4)). Let us next make this precise.

In view of equations (3.9)-(3.10), let us also partition $\tilde{G}$ and $\tilde{G}_{0}$ as

$$
\tilde{G}=\left(\begin{array}{c|c}
\lambda & u^{T} \\
\hline u & \mathscr{G}
\end{array}\right), \quad \tilde{G}_{0}=\left(\begin{array}{c|c}
\lambda_{0} & u_{0}^{T} \\
\hline u_{0} & \mathscr{G}_{0},
\end{array}\right),
$$

where $\lambda: I \rightarrow \mathbb{C}, u: I \rightarrow \mathbb{C}^{2 \times 1}$, and $\mathscr{G}: I \rightarrow \mathbb{C}^{2 \times 2}$ are functions, and $\lambda_{0} \in \mathbb{C}, u_{0} \in$ $\mathbb{C}^{2 \times 1}, \mathscr{G}_{0} \in \mathbb{C}^{2 \times 2}$ are initial values. That $\operatorname{Im} \tilde{G}_{0}$ is positive definite means in terms of $\lambda_{0}, u_{0}, \mathscr{G}$ that

$$
\operatorname{Im} \lambda_{0}>\operatorname{Im} u_{0}^{T}\left(\operatorname{Im} \mathscr{G}_{0}\right)^{-1} \operatorname{Im} u_{0}, \quad \operatorname{Im} \mathscr{G}_{0} \text { positive definite. }
$$

This follows using a Shur complement on $\operatorname{Im} \tilde{G}$ and the fact: if $S$ is invertible, then $A$ is positive definite if and only if $S^{T} A S$ is positive definite.

With above notation, equation (4.1) decomposes into three differential equations

$$
\begin{aligned}
\lambda^{\prime} & =-u^{T} u, \\
u^{\prime} & =-\mathscr{G} u, \\
\mathscr{G}^{\prime}+\mathscr{G}^{2}-\mathscr{R} & =0,
\end{aligned}
$$

with initial values $\lambda(0)=\lambda_{0}, u(0)=u_{0}, \mathscr{G}(0)=\mathscr{G}_{0}$. If we write $\mathscr{G}=\mathscr{G}_{r}+i \mathscr{G}_{i}$, where $\mathscr{G}_{r}, \mathscr{G}_{i}$ are real, then equation (4.4) reads

$$
\begin{aligned}
\mathscr{G}_{r}^{\prime}+\mathscr{G}_{r}^{2}-\mathscr{R} & =\mathscr{G}_{i}^{2}, \\
\mathscr{G}_{i}^{\prime}+\mathscr{G}_{r} \mathscr{G}_{i}+\mathscr{G}_{i} \mathscr{G}_{r} & =0 .
\end{aligned}
$$

Physically, $\operatorname{Im} \lambda$ describes how rapidly the Gaussian beam decays in the direction of propagation, and $\operatorname{Im} \mathscr{G}$ describes its decay in the transversal plane. If $\operatorname{Im} u=0$, the decay of the Gaussian beam is determined by an ellipsoid aligned with one axis along $\dot{c}$. Equation (4.4) shows that we can solve $\mathscr{G}$, that is $\tilde{G}_{\alpha \beta}$, without solving $u$ or $\lambda$.

Proposition 4.1. Let $\tilde{G}$ be a solution to equation (4.1) decomposed as above.

1. Pointwise $u=0$ if and only if $\dot{c}=(1,0,0)$ is an eigenvector for $\tilde{G}$. In this case, $\lambda$ is the eigenvalue corresponding to $\dot{c}$.

2. If $u_{0}=0$, then $u$ is zero for all $t$, and $\lambda(t)=\lambda_{0}$ is constant.

Proof. Claim (i) is elementary. Claim (ii) follows by solution formula for equations (4.3). 
Let us next give a coordinate invariant formulation of projection $G \mapsto \mathscr{G}$. For this purpose, let $\Pi=\Pi_{j}^{i} \frac{\partial}{\partial x^{i}} \otimes d x^{j}$ be the tensor determined by coefficients

$$
\begin{aligned}
\Pi_{i}^{j} & =g_{i a} C^{a j} \\
& =\delta_{i}^{j}-p_{i} \dot{c}^{j} .
\end{aligned}
$$

Then generally $\Pi \circ \Pi=\Pi$,

$$
v=\Pi(v)+g(\dot{c}, v) \dot{c}, \quad v \in T_{c(t)} M,
$$

and ker $\Pi=\operatorname{span}(\dot{c})$. Thus $\Pi$ is the projection operator mapping $T_{c(t)} M$ onto the orthogonal complement of $\dot{c}(t)$. For a 2-tensor $B=B_{i j} d x^{i} \otimes d x^{j}$, let $\Pi B \Pi^{T}=\Pi_{i}^{a} B_{a b} \Pi_{j}^{b} d x^{i} \otimes d x^{j}$ whence the matrix representing $\Pi B \Pi^{T}$ in Fermi coordinates is $\tilde{\Pi} \tilde{B} \tilde{\Pi}^{T}$, where $\tilde{\Pi}=\left(\tilde{\Pi}_{i}^{j}\right)_{i j}=$ $\left(\tilde{C}^{i j}\right)_{i j}$. For example, the matrix representing $\Pi G \Pi^{T}$ in Fermi coordinates is

$$
\tilde{\Pi} \tilde{G} \tilde{\Pi}^{T}=\operatorname{diag}(0, \mathscr{G}) \text {. }
$$

Proposition 4.2 (Riccati equation in transversal plane). Let $G_{0}$ be a symmetric initial value with positive definite imaginary part. If tensor $G$ is a solution to equation (1.1) with initial value $G_{0}$, then

$$
F=\Pi G \Pi^{T}
$$

is also a solution to equation (1.1), but with initial value $\Pi G_{0} \Pi^{T}$. Conversely, if $G$ and $F$ are solutions from initial values $G_{0}$ and $\Pi G_{0} \Pi^{T}$, respectively, then $F=\Pi G \Pi^{T}$.

Proof. Since equation (1.1) is a tensor equation, it suffices to prove the claim in Fermi coordinates. Thus, by assumption $\tilde{G}$ solves equation $(4.1)$ with $\tilde{G}(0)=\tilde{G}_{0}$. Then $\mathscr{G}$ satisfies equation (4.4) with $\mathscr{G}(0)=\mathscr{G}_{0}$. If $F=\Pi G \Pi^{T}$, then $F_{0}=\Pi G_{0} \Pi^{T}$, and $\tilde{F}=$ $\operatorname{diag}(0, \mathscr{G})$ satisfies equation $(4.1)$. For the second claim, let us first note that in Fermi coordinates, $\tilde{F}=\operatorname{diag}(0, \mathscr{F})$ for some $2 \times 2$ matrix $\mathscr{F}$. This follows from equations (4.2)(4.3), and their solution formulas. Let $\tilde{C} \tilde{G} \tilde{C}=\operatorname{diag}(0, \mathscr{G})$. Then $\mathscr{F}$ and $\mathscr{G}$ share the same initial value, which is symmetric and has positive definite imaginary part. Since, in addition, $\mathscr{F}$ and $\mathscr{G}$ both solve equation (4.4), we have $\mathscr{F}=\mathscr{G}$. This gives $\tilde{F}=$ $\operatorname{diag}(0, \mathscr{F})=\operatorname{diag}(0, \mathscr{G})=\tilde{C} \tilde{G} \tilde{C}$.

\subsection{Shape operator of surfaces $F_{t}$ and $S_{t}$}

Next we calculate shape operators of surfaces $F_{t}$ and $S_{t}$. This will be done using Lemma 4.3 and Lemma 4.4. To formulate these lemmas, we need the Legendre transformation $\sharp: T^{*} M \rightarrow T M$ defined as $\sharp: \alpha_{i} d x^{i} \mapsto g^{i j} \alpha_{i} \frac{\partial}{\partial x^{j}}$. Let us also write $\alpha^{\sharp}=\sharp(\alpha)$.

Lemma 4.3 (Shape operator for an equipotential surface). Let $p \in M$. Suppose $f: M \rightarrow$ $\mathbb{R}$ is a smooth function with $d f_{p} \neq 0$, whence

$$
S=\{x \in M: f(x)=f(p)\}
$$

is a hypersurface passing trough $p$. Suppose further that $g\left((d f)^{\sharp},(d f)^{\sharp}\right)=1$ at $p$, and $S$ is oriented with a unit normal $n$ such that $n=d f^{\sharp}$ at $p$. Then the shape operator of $S$ at $p$ equals

$$
L(v)=\pi \circ \sharp \circ D_{v}(d f), \quad v \in T_{p} S,
$$

where $\pi: T_{p} M \rightarrow T_{p} S$ is the projection $\pi(v)=v-g(v, n) n$ for $v \in T_{p} M$. 
Proof. Suppose $E \in \mathfrak{X}(M)$ is vector field

$$
E(x)=(d f)^{\sharp}(x), \quad x \in M,
$$

whence $g(E, E)=1$ at $p \in M$, and $\hat{n}=E / \sqrt{g(E, E)}$ is an extension of a correctly oriented unit normal on $S$. Suppose $v \in T_{p} M$. Then $D_{v}(f X)=v(f) X+f D_{v}(X)$ for $X \in \mathfrak{X}(M)$, and $D_{v}\left(\alpha^{\sharp}\right)=\sharp \circ D_{v}(\alpha)$ for $\alpha \in \Omega^{1}(M)$. These identities together with $\pi \circ L=L$ imply the result.

Lemma 4.4 (Shape operator in Fermi coordinates). Suppose $f$ and $S$ are as in the previous lemma, $c$ is a geodesic parametrized with respect to pathlength such that $\dot{c}(t)=(d f)^{\sharp}(p)$, and $(s, \mu)$ are Fermi coordinates around $c$. Then the shape operator of $S$ at $c(t)$ is

$$
L(v)=\sum_{\alpha, \beta=2}^{3} \tilde{v}^{\alpha} \frac{\partial^{2} f}{\partial \mu^{\alpha} \partial \mu^{\beta}}(p) \frac{\partial}{\partial \mu^{\beta}}, \quad v \in T_{p}(S) .
$$

Theorem 4.5 (Shape operator of surface $F_{t}$ ). Let phase front $F_{t}$ be oriented with unit normal $n$ such that $n_{c(t)}=\dot{c}(t)$. Then the shape operator of $F_{t}$ at $c(t)$

$$
L: T_{c(t)} F_{t} \rightarrow T_{c(t)} F_{t}
$$

is given by

$$
L(v)=v^{i}\left(\Pi \operatorname{Re} G \Pi^{T}\right)_{i j} g^{j k} \frac{\partial}{\partial x^{k}}, \quad v \in T_{c(t)} F_{t},
$$

or in Fermi coordinates,

$$
L(v)=\sum_{\alpha, \beta=2}^{3} \tilde{v}^{\alpha} \operatorname{Re} \tilde{G}_{\alpha \beta} \frac{\partial}{\partial \mu^{\beta}}, \quad v \in T_{c(t)} F_{t} .
$$

Proof. We only need to prove the claim in Fermi coordinates. Let us fix $t$, whence $F_{t}=\{x \in M: f(x)=f(c(t))\}$ for $f(x)=\operatorname{Re} \theta(x, t)$. Since $(d f)^{\sharp}(c(t))=\dot{c}(t)$, Lemma 4.4 implies that

$$
L(v)=\sum_{\alpha, \beta=2}^{3} \tilde{v}^{\alpha} \frac{\partial^{2} f}{\partial \mu^{\alpha} \partial \mu^{\beta}} \frac{\partial}{\partial \mu^{\beta}}, \quad v \in T_{c(t)} F_{t} .
$$

By the chain rule we have $\frac{\partial^{2} f}{\partial \mu^{\alpha} \partial \mu^{\beta}}=\frac{\partial^{2} f}{\partial x^{i} \partial x^{j}} \frac{\partial x^{i}}{\partial \mu^{\alpha}} \frac{\partial x^{j}}{\partial \mu^{\beta}}+\frac{\partial f}{\partial x^{i}} \frac{\partial^{2} x^{i}}{\partial \mu^{\alpha} \partial \mu^{\beta}}$. Evaluating at $\mu=0$ and using equation (3.7) yields $\frac{\partial^{2} f}{\partial \mu^{\alpha} \partial \mu^{\beta}}(t, 0)=\operatorname{Re} \tilde{G}_{\alpha \beta}(t)$.

In the Euclidean inner product, the shape operator vanishes on hyperplanes. Intuitively this means that the Euclidean inner product sees no shape on a hyperplane. The next proposition gives an analogous result for $g$ and $S_{t}$.

Proposition 4.6 (Shape operator of surface $S_{t}$ ). The curve $c$ is orthogonal to $S_{t}$, and the shape operator on $S_{t}$ (with respect to $g$ ) is identically zero at $c(t)$. 
Proof. The first claim follows since $T_{c(t)} S_{t}=\operatorname{span}\left\{\frac{\partial}{\partial \mu^{2}}, \frac{\partial}{\partial \mu^{3}}\right\}$. The second claim follows from Lemma 4.4 as $S_{t}=\{(s, \mu): s-t=0\}$.

Let us recall Gauss' lemma in Riemannian geometry [3, 19]. It states that if $B$ is a geodesic ball around a point $p$, then geodesics from $p$ will intersect the boundary of $B$ orthogonally. In the setting of Gaussian beams, a geodesic ball around a point represents the area of influence. In time $t$, no Gaussian beam can propagate outside a geodesic ball of radius $t$. Proposition 2.1 and 4.6 state that for small $t$, surfaces $F_{t}$ and $S_{t}$ are tangential to the geodesic sphere of radius $t$.

\section{Gaussian beams in Euclidean geometry}

Let us consider the case when $g$ is Euclidean, that is, $g=\delta_{i j} d x^{i} \otimes d x^{j}$, and the geodesic is $t \mapsto(t, 0,0)$. Then Fermi coordinates $(s, \mu)$ coincide with Euclidean coordinates; $s=x^{1}$, $\mu^{2}=x^{2}$, and $\mu^{3}=x^{3}$.

\subsection{Geometry of surface $F_{t}$}

Let us study the shape of $F_{t}$ for $t=0$. For simplicity, let us assume that $\operatorname{Re} G_{1 i}=0$ for all $i, c(t)=0$, and $\theta$ is a second order polynomial in $z$. The equation for $F_{t}$ then reads

$$
s=-\frac{1}{2} \operatorname{Re} G_{\alpha \beta} \mu^{\alpha} \mu^{\beta} .
$$

If we assume that $\operatorname{Re} G_{11}$ is non-zero, then one obtains a second order equation for $t$. Of the two solutions, one must be excluded as it does not pass through $c(t)$, and if one approximates $\sqrt{1+x}=1+x / 2$ in the other, it will coincide with the above solution. Figure 1 shows how $F_{t}$ depends on the definiteness of $\operatorname{Re} \mathscr{G}$.
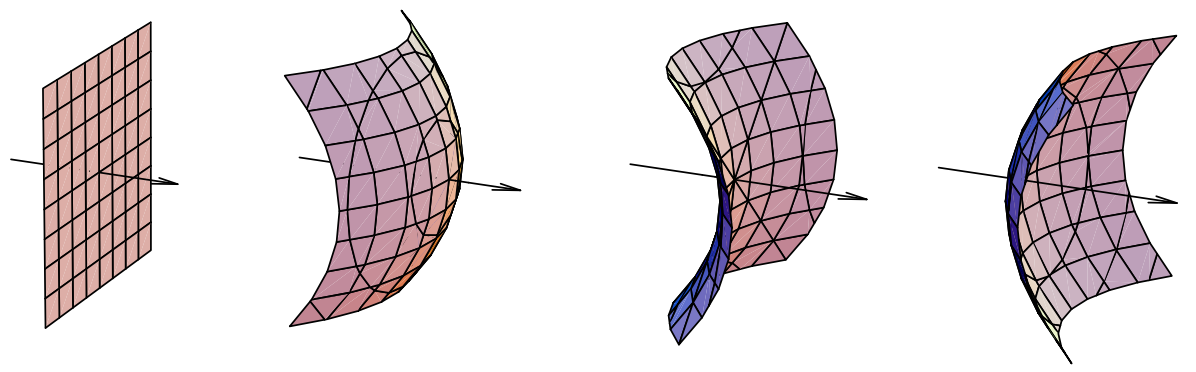

Figure 1. Phase front $F_{t}$ in cases $\operatorname{Re} \mathscr{G}=0, \operatorname{Re} \mathscr{G}$ positive definite, $\operatorname{Re} \mathscr{G}$ semi-definite, and $\operatorname{Re} \mathscr{G}$ negative definite. The arrow shows direction of propagation.

\subsection{Explicit solution to $\mathscr{G}$}

In Euclidean geometry, $R=0$, and the solution to equation (4.1) is

$$
G(t)=G_{0}\left(I+t C G_{0}\right)^{-1}, \quad C=\operatorname{diag}(0,1,1),
$$


or, in partitioned form,

$$
\begin{aligned}
\lambda(t) & =u_{0}^{T} \mathscr{G}_{0}^{-1}\left(I+\mathscr{G}_{0} t\right)^{-1} u_{0}+k, \\
u(t) & =\left(I+\mathscr{G}_{0} t\right)^{-1} u_{0}, \\
\mathscr{G}(t) & =\mathscr{G}_{0}\left(I+\mathscr{G}_{0} t\right)^{-1},
\end{aligned}
$$

where

$$
k=\lambda_{0}-u_{0}^{T} \mathscr{G}_{0}^{-1} u_{0}=\operatorname{det} \mathscr{G}_{0}^{-1} \operatorname{det} G_{0} .
$$

The expressions for $G$ and $\mathscr{G}$ can be derived using the standard solution method for a matrix Riccati equation as outlined in Section 2.3. However, it is maybe easiest to just verify these by direct substitution using matrix identity $\frac{d A^{-1}}{d t}=-A^{-1} \frac{d A}{d t} A^{-1}$, and the fact that $A$ and $(I+A)^{-1}$ commute. Inverse $\left(I+\mathscr{G}_{0} t\right)^{-1}$ exists by Lemma 2.3 , and $\left(I+t C G_{0}\right)^{-1}$ exists since $\operatorname{det}\left(I+t C G_{0}\right)=\operatorname{det}\left(I+\mathscr{G}_{0} t\right)$. Let $J=\left(\begin{array}{cc}0 & 1 \\ -1 & 0\end{array}\right)$. If $B$ is an invertible $2 \times 2$ symmetric matrix, then $B^{-1}=\frac{-1}{\operatorname{det} B} J B J$. Thus

$$
\left(I+\mathscr{G}_{0} t\right)^{-1}=\frac{I-J \mathscr{G}_{0} J t}{\operatorname{det}\left(I-J \mathscr{G}_{0} J t\right)} .
$$

From this expression it follows that $\lambda(t), u(t)$, and $\mathscr{G}(t)$ decay as $1 / t$. Thus, if $I=\mathbb{R}$, then

$$
\lim _{t \rightarrow \infty} \lambda(t)=\operatorname{det} \mathscr{G}_{0}^{-1} \operatorname{det} G_{0}, \quad \lim _{t \rightarrow \infty} u(t)=0, \quad \lim _{t \rightarrow \infty} \mathscr{G}(t)=0,
$$

In particular, at infinity the Gaussian beam is infinitely wide $(\mathscr{G}=0)$

Example 5.1 (Eigenvalues of $\operatorname{Re} \mathscr{G}$ and $\operatorname{Im} \mathscr{G}$ ). Let us study solutions from initial values of the form

$$
\mathscr{G}_{0}=\operatorname{diag}(a+i, c+i),
$$

where $a, c$ are real eigenvalues of $\operatorname{Re} \mathscr{G}$. In this case, the Riccati equation decouples into two scalar equations. Figure 2 shows the eigenvalues of $\operatorname{Re} \mathscr{G}$ and $\operatorname{Im} \mathscr{G}$ for six values for $a$ and $c$. The signum-type function in the plots will be studied in Section 5.3.

In the plots we can see three phenomena. First, in all plots the magnitudes of the eigenvalues tend to zero. In other words, for large $t$, the phase front will resemble more and more a plane. Secondly, even if initial value $\operatorname{Re} \mathscr{G}_{0}$ is semi-definite or zero, the solution $\operatorname{Re} \mathscr{G}$ always tends to a positive definite matrix. This suggests that the solution is unstable if $\operatorname{Re} \mathscr{G}$ is not positive definite. This is also physically reasonable. In free space, the phase surface should look like a sphere, not as a hyperbola (see Figure 1). Lastly, eigenvalues of $\operatorname{Re} \mathscr{G}$ and $\operatorname{Im} \mathscr{G}$ are coupled.

\subsection{When is the phase front within the area of influence?}

Let us write the general initial value $\mathscr{G}_{0}$ as

$$
\mathscr{G}_{0}=\left(\begin{array}{ll}
A & B \\
B & C
\end{array}\right)=\left(\begin{array}{ll}
a & b \\
b & c
\end{array}\right)+i\left(\begin{array}{ll}
\alpha & \beta \\
\beta & \gamma
\end{array}\right),
$$



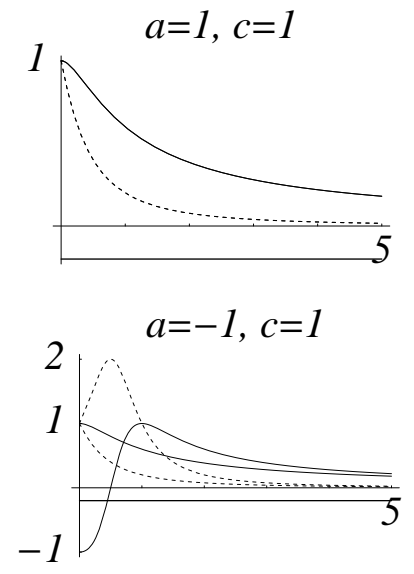
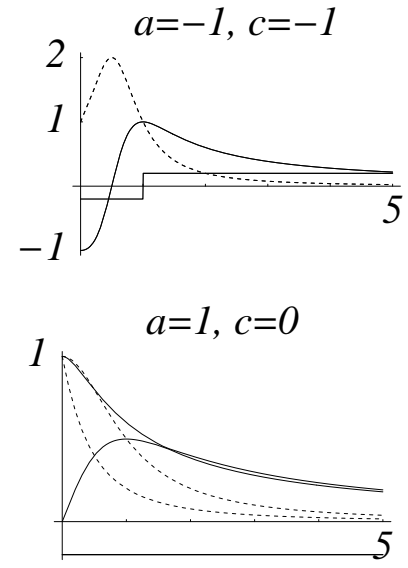
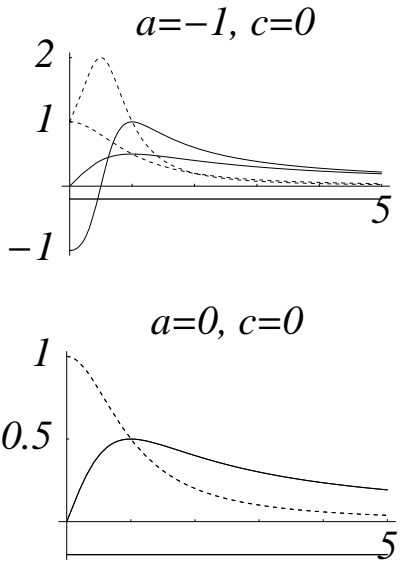

Figure 2. Eigenvalues of $\operatorname{Re} \mathscr{G}$ (solid lines) and $\operatorname{Im} \mathscr{G}$ (dashed lines) for $t=0, \ldots, 5$ from initial value (5.1). The signum-type functions is described in Section 5.3.

where $A, B, C$ are complex numbers, and $a, b, c, \alpha, \beta, \gamma$ are real. Computer algebra gives the following expressions for $K$ and $S$,

$$
\begin{aligned}
K(t) & =\frac{C_{4} t^{2}+\frac{1}{2} C_{3} t+C_{0}}{C_{4} t^{4}+C_{3} t^{3}+C_{2} t^{2}+C_{1} t+1}, \\
S(t) & =\frac{1}{2} \frac{2 C_{4} t^{3}+\frac{3}{2} C_{3} t^{2}+C_{2} t+\frac{1}{2} C_{1}}{C_{4} t^{4}+C_{3} t^{3}+C_{2} t^{2}+C_{1} t+1},
\end{aligned}
$$

where

$$
\begin{aligned}
& C_{0}=\operatorname{det} \operatorname{Re} \mathscr{G}_{0}, \\
& C_{1}=2 \operatorname{trace} \operatorname{Re} \mathscr{G}_{0}, \\
& C_{2}=\left(\operatorname{trace}\left(\operatorname{Re} \mathscr{G}_{0}\right)\right)^{2}+2 \operatorname{det} \operatorname{Re} \mathscr{G}_{0}+\left(\operatorname{trace}\left(\operatorname{Im} \mathscr{G}_{0}\right)\right)^{2}-2 \operatorname{det} \operatorname{Im} \mathscr{G}_{0}, \\
& C_{3}=2\left(c|A|^{2}+a|C|^{2}-\operatorname{Re}\left(B^{2} \operatorname{trace}\left(\mathscr{G}_{0}^{*}\right)\right)\right), \\
& C_{4}=\left|\operatorname{det} \mathscr{G}_{0}\right|^{2},
\end{aligned}
$$

and $M^{*}$ is the complex conjugate of a matrix $M$. These expressions can be derived either using Proposition 2.2, or from the eigenvalues of $\mathscr{G}$ and using Theorem 4.5. It follows that $K$ decays as $1 / t^{2}$, and $S$ decays as $1 / t$. Thus for large $t$ the Gaussian curvature of $F_{t}$ (at $c(t)$ ) approaches $1 / t^{2}$ which is the curvature for a sphere of radius $t$. The sphere with center $c(0)$ and radius $t$ represents the area of influence; in time $t$, a signal can propagate at most a distance of $t$ units. As the phase front describes how the field propagates, it would be natural to assume that near $c(t)$, phase front $F_{t}$ is contained inside this sphere. Suppose that $\operatorname{Re} G_{1 i}=0$ for all $i$. Then the above condition translates into

$$
\kappa_{1}(t)>\frac{1}{t}, \quad \kappa_{2}(t)>\frac{1}{t}
$$

for principal curvatures $\kappa_{1}, \kappa_{2}$. These inequalities are only reasonable for large $t$. The signum-type function plotted in Figure 2 indicates when condition (5.2) holds. When the 
function is positive the condition holds and when negative the condition does not hold. Let us point out that $G(t)$ in $a=c=1$ is identical to $G(t+1)$ in $a=c=-1$. However, condition (5.2) is not translationally invariant, so the signum functions do not coincide. Schematically condition (5.2) is illustrated in Figure 3. In the left figure, the condition holds. In other words, phase fronts are contained within the area of influence. The right figure shows phase fronts where $\operatorname{Re} \mathscr{G}$ are positive definite, but the condition does not hold.
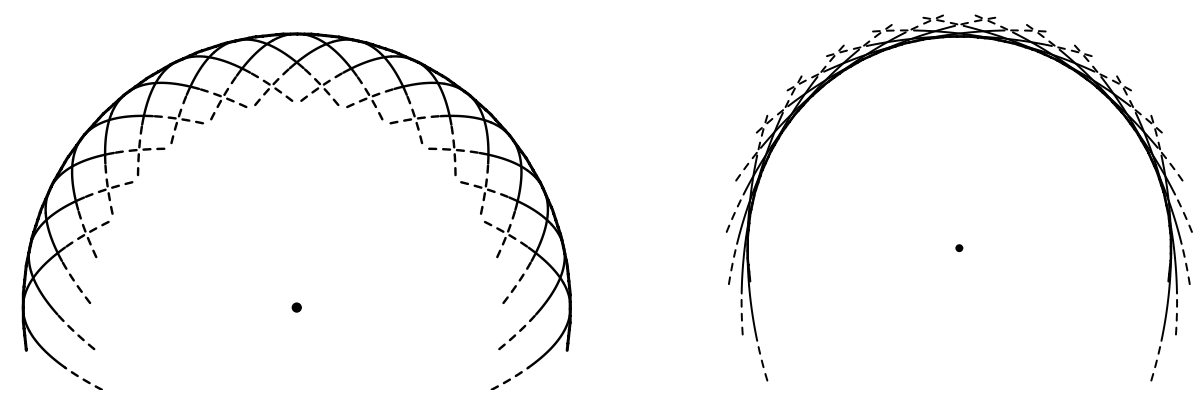

Figure 3. A $2 D$ illustration of phase fronts in cases $K(t)>1 / t^{2}$ (left) and $K(t)<1 / t^{2}$ (right).

Suppose $B=0$. Then matrix Riccati equation (4.4) decomposes into two scalar equations, and in this case we have

$$
\kappa_{1}(t)=\frac{a+\left(a^{2}+\alpha^{2}\right) t}{(1+a t)^{2}+(t \alpha)^{2}}, \quad \kappa_{2}(t)=\frac{c+\left(c^{2}+\gamma^{2}\right) t}{(1+c t)^{2}+(t \gamma)^{2}},
$$

and condition (5.2) takes the simple form: $-1>a t,-1>c t$. Thus, for large $t$, condition (5.2) holds if and only if $a, c<0$. It follows that in Figure 2, condition (5.2) will only be satisfied for $a=c=-1$. In addition, by slightly perturbing initial values $(0,0),(-1,0)$, and $(0,-1)$ these too satisfy the condition.

Acknowledgments. This work has been funded by the Graduate School of Applied Electromagnetism and the Institute of Mathematics at the Helsinki University of Technology. I would like to thank my Phd. instructor K. Peltonen for suggesting this topic and for helpful discussions, and Professor A. P. Kachalov for pointing out references [1, 2].

\section{References}

[1] Babich V M and Pankratova T F, The discontinuities of the Green's function for a mixed problem for the wave equation with variable coefficients, Probl. Math. Phys. 6 (1973), 9-27. [in Russian]

[2] BABICH V M and Ulin V V, The complex space-time ray method and "quasiphotons", Zap. Nauchn. Semin. LOMI 117 (1981), 5-12. [in Russian]

[3] Buchtaru I and Slawinski M A, Generalized orthogonality between rays and wavefronts in anisotropic inhomogeneous media, Nonlinear Anal.-Real World Appl. 6 (2005), 111-121.

[4] Červený V, Seismic Ray Theory, Cambridge University Press, 2001. 
[5] DAhL M, Propagation of electromagnetic Gaussian beams using Riemannian geometry, Progress Electromagn. Res. 60 (2006), 265-291.

[6] Eisenhart L P, Riemannian geometry, Princeton University Press, 1949.

[7] Gray A, Tubes, Addison-Wesley Publishing Company, 1990.

[8] Jost J, Riemannian geometry and geometric analysis, Springer, 1998.

[9] Kachalov A P, Gaussian beams, Hamilton-Jacobi equations, and Finsler geometry, Zap. Nauchn. Sem. S.-Peterburg. Otdel. Mat. Inst. Steklov. (POMI) 297 (2003), 66-92, [in Russian]; J. Math. Sci. 127 (2005), 2374-2388. [in English]

[10] Kachalov A P, Nonstationary electromagnetic Gaussian beams in nonhomogeneous anisotropic medium, J. Math. Sci. 111 (2002), 3667-3677.

[11] Kachalov A P, Gaussian beams for Maxwell Equations on a manifold, J. Math. Sci. 122 (2004), 3485-3501.

[12] Kachalov A, Kurylev Y and Lassas M, Inverse Boundary Spectral Problems, Chapman \& Hall/CRC, 2001.

[13] Kachalov A and Lassas M, Gaussian beams and Inverse Boundary Spectral Problems, in New analytic and geometric methods in inverse problems, Springer, Editors: Bingham K, Kurylev Ya V and Somersalo E, 2004, 127-163.

[14] Kachalov A P and Popov M M, Application of the Gaussian beam method to elasticity theory, Geophysics J. Roy. Astron. Soc. 81 (1985), 205-214.

[15] Manasse F K and Misner C W, Fermi normal coordinates and some basic concepts in differential geometry, J. Math. Phys. 4 (1963), 735-745.

[16] Popov M M, Ray theory and Gaussian beams method for geophysicists, EDUFBA, 2002.

[17] Ralston J, Gaussian Beams and the Propagation of Singularities, Stud. Partial Diff. Eq., MAA Studies in Mathematics 23 (1982), 206-248.

[18] Shen Z, Lectures on Finsler Geometry, World Scientific, 2001.

[19] SpIVAK M, A comprehensive introduction to differential geometry, Vol. 1, Publish or Perish, Inc., 1979.

[20] SPIVAK M, A comprehensive introduction to differential geometry, Vol. 3, Publish or Perish, Inc., 1979. 